\title{
Models of Extended Electron with a Point Charge
}

\author{
Sovan Ghosh \\ Post Graduate Department of Physics, Vijaya College, Bangalore - 560004, Karnataka \\ E-mail: gsovan@gmail.com
}

https://doi.org/10.54162/SD01-25201/07

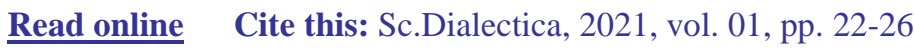

Keywords: Electron, Point-like charge, Zero point fluctuation

\begin{abstract}
The discovery of the electron insisted and inspired a number of theoretical predictions which are formulated in terms of models. A lot of models in wide variety were proposed for more than a century time. An attempt of a mini-review of them is taken here. But as a shorter version here only extended models with point charge are discussed instead of all.
\end{abstract}

\section{Introduction}

Following the discovery of the electron as the first subatomic particle in 1897 [1] several theoretical approaches were initialized to provide a better understanding of this enigmatic particle. But the structure of the electron is considerably a good topic though very recent research is advocating for a spherical electron [2] from the data of electric dipole moment of the same.

Depending on the properties of the electron (for the most current data one can have a look at recent volume of Particle Data Group) numbers of models of the electron [1-35, 37, 39-64] have been proposed theoretically over more than a century.

According to the experimental data the electron is a fundamental particle that does not decay into other particle and hence it is described as a point particle in the standard model of the particle physics. But with a non-zero definite mass, electric dipole moment, magnetic moment is it proper to label electron to be structure-less? As this question haunted physicists, logics, counter logics and models are over-poured in this subject area.

These models have been developed both in classical and quantum mechanical domain and some of them are at the boundary of the two and are known as semi-classical approaches. Crudely the models of the electron are either point-particle models or extended models. Advancing further
P Lancini classified all electron models in three types: Point-models [11-14], Extended models [3, 5, 15-20] and Extended models with point charge [7-9]. Some other models are also there which does not fall in these three kinds and they are chiefly sub-structure models. Lepton and quark sub-structure models are proposed in good number of articles [21-26].

Also there are some other theories, which deal with the extra dimensions or above the known four dimensions [9, 26-27] and they provide the picture of the electron in their own ways. Gravity as a zero point fluctuation [28] is an important model.

To discuss all of them would be a very lengthy discussion. So here we will restrict ourselves only within the last kind.

\section{Discussion}

Extended models with point-like charge

\section{(i) Bunge model}

Without executing Foldy-Wouthuysen transformation [3638], a mean position operator with a smooth motion is derived by M. Bunge to illustrate the picture of the electron [7]. This picture represents the extended electron of the Compton size with a point charge and a distribution of mass. This is a Compton-sized model with a point charge and distributed mass. The mean-position operator was provided by L. L. Foldy and S. A. Wouthuysen. This operator is named by Bunge as the center-of-mass operator. The operator provided by Foldy-Wouthuysen is not oscillatory in nature and the corresponding time derivative is proportional to the momentum. This is read as [7]

$$
x^{\prime}=e^{-i s} x e^{i s},
$$

where, $x$ is the point where charge is concentrated. The displacement operator proposed by Bunge is

$T=e^{i s}$,

where, $s=\frac{\Lambda}{2} \sum_{0}^{3}{ }_{\mu} \gamma^{\mu} \frac{\partial}{\partial x^{\mu}}, \mu=0,1,2,3$ 
Here $\Lambda=\frac{\hbar}{m_{0} c}$ is the Compton radius,

and $\gamma=\left|\begin{array}{cc}0 & \sigma \\ -\sigma & 0\end{array}\right|$

A model of the electron within the frame of the standard representation of the one particle theory is represented here. Extended structure and point particle notion are merged in this picture. Here Lorentz force has been employed to the terminus of the dancing vector and that depicts the charge of Dirac's electron [6] at that point. This oscillation is around the mean position co-ordinate with amplitude of the Compton radius, $\Lambda$. The mass of the body is assumed to be spread over a region of dimensions of a Compton wavelength or $\Lambda$.

The trajectory of the movement of the electron and the details of the dynamics are not absolutely shaped by Bunge in greater depth. But this endeavor of him was completely out of the box of classical approaches to define this subatomic particle as well as this respected the experimentally measured facts regarding electric dipole moment, magnetic moment and the different sizes of the electron.

\section{(ii) Relativistic Spinning Sphere Model (RSS)}

A semi-classical structure of the 'enigmatic electron' is portrayed by relativistic spinning sphere model [8, 39-40] of the electron. The spectroscopic properties of the electron at the first order of the fine structure constant, $\alpha$ is theorised in RSS model.

Framing the characteristic features, the sphere is considered to be made of the non-interacting but rigid mechanical mass along with the point charge e, residing at the equatorial zone of the sphere. This spherical model successfully transforms under the Lorentz transformation too. The observed electron spin, the total quantum-mechanical spin and the accurate gyromagnetic ratio are reproduced exactly by this model. MacGregor has shown the context of the different energies corresponding to the electron structure. The spinning mass is taken here as $m_{s}=\frac{3}{2} m_{0}$, where $\mathrm{m}_{0}$ is the rest mass of the electron. Starting with the relationship between the mass and the spin angular momentum of the electron, the RSS model is developed.

MacGregor considered the well-known facts about the electron to build up the relativistic spinning sphere model of the electron. They are: a) the electron is spinning, b) the spin is quantized, c) the spin is $J=\frac{1}{2} \hbar$ and it is very large with respect to the mass of the electron, d) the spherical shape of the electron is not changed due to relativistic rotation.
As the relativistic rotation incorporates the increase in mass, the rest mass $m_{0}$ of an element of ring is modified according to

$$
m(r)=\frac{m_{0}(r)}{\sqrt{1-\omega^{2} r^{2} / c^{2}}},
$$

where. $\omega$ is the angular velocity of the spinning ring and $r$ is the distance of the element from the axis of rotation. For a sphere with radius $\mathrm{R}$, the mass-density of the sphere is figured out as,

$$
D=\frac{m(r)}{V_{\text {sphere }}}=\frac{3 m_{0}(r)}{4 \pi R^{3} \sqrt{1-\omega^{2} r^{2} / c^{2}}},
$$

where, $V_{\text {sphere }}$ is the volume of the entire sphere. To get the relativistic mass of a spinning sphere, it is easier to explore the axially centred cylindrical mass elements. The volume of the cylindrical element becomes

$$
V(r)=4 \pi \sqrt{R^{2}-r^{2}} r d r,
$$

where, $R$ is the radius of the sphere and $r$ is the distance of the element from the axis of rotation. So the spinning mass of the element of the ring can be calculated as

$$
m_{s}=\frac{3 m_{0}}{R^{3}} \sqrt{\frac{R^{2}-r^{2}}{1-\omega^{2} r^{2} / c^{2}}} r d r .
$$

Therefore for the entire sphere, the spinning mass is

$$
M_{s}=\frac{3 M_{0}}{R^{3}} \int_{0}^{R} \sqrt{\frac{R^{2}-r^{2}}{1-\omega^{2} r^{2} / c^{2}}} r d r,
$$

where, $M_{0}$ is the non-spinning mass. With the equatorial velocity $c$ of the spinning sphere, the angular velocity attains the highest value without violating the special theory of relativity as

$$
\omega=\frac{c}{R} .
$$

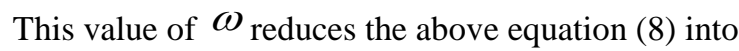

$$
M_{s}=\frac{3 M_{0}}{R^{3}} \int_{0}^{R} R r d r
$$

which implies

$$
M_{s}=\frac{3}{2} M_{0} \text {. }
$$

MacGregor has established the model keeping the theory of relativity in his mind. That helped him to write down separately the conditions of non-rotating and the rotating frames. The relativistic moment of inertia of the spinning sphere about the axis of rotation comes out as

$I=\frac{3}{4} m_{0} R^{2}=\frac{1}{2} m R^{2}$.

In the conclusion, it is shown that the mechanical mass constitutes $99.9 \%$ of the observed mass. 


\section{(iii) Dynamical spinning sphere model}

A classical model, called dynamical spinning sphere model, for a spinning electron is proposed by M. Rivas in the framework of kinematical formalism [9, 41-42]. The dynamics of the system is expressed in terms of some arbitrary evolution parameter $\tau$ though the Lagrangian is independent of $\tau$. In the generalized Lagrangian, some kinematical variables will be the time derivatives of some other kinematical variables depending on the nature of the higher order derivatives. Thus the dynamical variables will no longer be of second order, rather fourth order or of higher, which advocate the condition for the charge position of a spinning particle.

In this formalism, author has made good use of Galilei group of space-time transformation [41] to represent the dynamics of the elementary particles. The action of a group element $g \equiv(b, \vec{a}, \vec{v}, \vec{\alpha})$ on a space-time point $x \equiv(t, \vec{r})$, is represented by $x^{\prime}=g x . \vec{a}$ is the space parameter and $\mathrm{b}$ represents the time parameter whereas the velocity parameter is $\vec{v}$ and $\vec{\alpha}$ is dimensionless. The corresponding expression for the $x^{\prime}$ is given as

$$
x^{\prime}=\exp (b H) \exp (\vec{a} \cdot \vec{P}) \exp (\vec{v} \cdot \vec{K}) \exp (\vec{\alpha} \cdot \vec{J}) x,
$$

where, $H, \vec{P}, \vec{K}$ and $\vec{J}$ are the generators of the Poincare group. This is a rotation of the point, followed by a pure Galilei transformation and a space and time translation.

The Lagrangian for the non-relativistic spinning elementary particle is given as

$$
L=T \dot{t}+\vec{R} \cdot \dot{r}+\vec{U} \cdot \dot{u}+\vec{V} \cdot \dot{\rho}
$$

and the functions are $T=\frac{\partial L}{\partial \dot{t}}, R_{i}=\frac{\partial L}{\partial \dot{r}^{i}}, U_{i}=\frac{\partial L}{\partial \dot{u}^{i}}$ and $V_{i}=\frac{\partial L}{\partial \dot{\rho}^{i}}$. In general, they will be the functions of the ten kinematical variables $(t, \vec{r}, \vec{u}, \vec{\rho})$ and the homogeneous functions of zero degree of the derivatives $(t, \dot{r}, \dot{u}, \dot{\rho})$. Similarly the relativistic condition is described as $L=T \dot{t}+\vec{R} \cdot \dot{r}+\vec{U} \cdot \dot{u}+\vec{W} \cdot \dot{w}$,

where, $T=\frac{\partial L}{\partial \dot{t}}, R_{i}=\frac{\partial L}{\partial \dot{r}^{i}}, U_{i}=\frac{\partial L}{\partial \dot{u}^{i}}$ and $W_{i}=\frac{\partial L}{\partial \omega^{i}}$. In general, they will be the functions of the ten kinematical variables $(t, \vec{r}, \vec{u}, \vec{\alpha})$ and the homogeneous functions of zero degree of the derivatives $(t, \dot{r}, \dot{u}, \dot{\alpha})$. Here, $t$ represents the time parameter, $\vec{r}$ represents the position, $\vec{u}$ represents the velocity of the particle as $u=c$ and the orientation is given by $\rightarrow$ $\alpha$. This model describes the magnetic moment and the spin of the electron in a better way. This is concerned with the orientation part and ultimately describes the spinning sphere model by a fourth order differential equation using Frenet-Serret differential equations [43].

The mass of the particle got less attention in this work and that is a weaker point left by this model, as the mechanical mass plays a crucial role in the electron's total energy. This model has been built more or less considering the Zitterbewewgung model of the electron. But if structural phenomena to be considered, then the limits of the motion should also be defined as well, which is not in this case.

\section{Concluding remarks}

Puzzling behavior of the electron kept on mocking us regarding our experimental limitation to explain a tiny electron in all aspects. In that respect the theory of the electron is colorful and advanced. Variety of approaches solidified the improvement of the understanding of those properties as well. Very recent finding of ACME Collaboration on the improved limit on the electric dipole moment of the electron [2] provided more confidence to the concept of a spherical electron and of course that provided a huge support to the extended electron model. Thus experimental evidences are advancing to unveil the shape of the enigmatic electron.

Though a spherical electron is advocated by the observed values of the ACME Collaboration [2] regarding the extended structure of the smallest fundamental particle yet known, the point charge strengthens the support for QED calculation and the Standard model of the elementary particles. The calculation of the magnetic moment of the electron can be done using extended structure with point charge comparatively faster than the magnetic moment calculation using QED. This magnetic moment calculation is shown in an article using modified relativistic spinning sphere model by the present author [64]. Similarly the magnetic moment calculation of the muon can also be done by alike expressions and that can help us to theorize the conceptual base to overcome the recent crisis of explanation regarding the anomalous magnetic moment of the muon [65-66].

\section{References}

[1] S. Weinberg, The Discovery of Subatomic Particles, UK: Cambridge University Press, 2003.

[2] ACME Collaboration, "Improved limit on the electric dipole moment of the electron", Nature, 2018, vol. 562, 355 , 
[3] F. Rohrlich, Classical Charged Particles, Massachusetts: Addison-Wesley Publishing Company, Inc. 1965.

[4] V. Simulik, Ed. "What is electron?",Canada: Apeiron, 2005.

[5]J. L. Jimenez and I. Campos, "Models of the classical electron after a century", Found. Phys. Lett. 1999, vol. 12, pp. 127-146.

[6] D. Lynden-Bell, "Relativistically spinning charged sphere", Phys. Rev. D., 2004, vol. 70, pp. 104021-1-14.

[7] M. Bunge, “A Picture of the Electron". Nuovo Cimento 1955, vol. 1, pp. $977-985$.

[8] M. H. MacGregor, The Enigmatic Electron, Dordrecht: Kluwer Academic Publishers, 1992.

[9] M. Rivas, "The dynamical equation of the spinning electron", J. Phys. A: Math. Gen, 2003, vol. 36, no. 16, pp. $4703-4715$.

[10] P. Lancini, "A Model of the Electron in a 6-Dimensional Spacetime", Found. Phys., 1999, vol. 29, no. 2, pp. 251 265.

[11] T. E .Phipps Jr., "Prospects for the Electron" in What is electron?, V. Simulik Ed., Apeiron, Canada 2005, pp.43-58.

[12] M. A. Visser, "A classical model for the electron", Phys. Lett. A, 1989, vol. 139 (3,4) pp. 99 - 102.

[13]S. M. Blinder, "Singularity-free electrodynamics for point charges and dipoles: a classical model for electron selfenergy and spin", Eur. J. Phys. 2003, vol. 24, pp. 271-275.

[14] W. C. Daywitt, "A massless-point-charge model for the electron”, Progress in Physics, 2010, vol. 2, pp.17-18.

[15] H.S. Allen, "The case for a ring electron". Proc. Phys. Soc. London, 1918, vol. 31 pp.49.

[16] A. H. Compton, "The size and the shape of the electron", Phys. Rev. 1919, vol. 14, no. 1, pp. 20-43.

[17] A. H. Compton, "The size and the shape of the electron", Phys. Rev. 1919, vol. 14, no. 3, pp. 247-259.

[18] D. L. Bergman and J. P. Wesley, "Spinning Charged Ring Model of Electron Yielding Anomalous Magnetic Moment", 1990, Gali. Elect. vol. 1, pp. 63-67.

[19] H. Torres-Silva, "Spin and relativity: a semiclassical model for electron spin", Ingeniare, Revista chilena de ingenieria, 2008, vol. 16, pp. 72-77.

[20] D. Lortz, A classical model for the extended electron I, Zeitschrift fur Naturforschung A., 2005, vol.60a, pp. 149157.
[21] H. Terazawa, "How many leptons and quarks" Phys. Rev. D, 1977, vol. 16, pp. $2373-2376$.

[22] H. Terazawa, "Subquark model of leptons and quarks", Phys. Rev. D, 1980, vol.22, pp. $184-199$.

[23] H. Terazawa, "The electron in the unified composite model of all fundamental particles and forces", in V. Simulik Ed. What is electron?, Canada: Apeiron 2005, pp. $29-42$.

[24] W. Krolikowski, "Towards a dynamic preon model", Acta Phys. Polo. 1980, vol. B11, pp. 431-438.

[25] J. C. Pati and A. Salam, "Lepton number as the fourth “color", Phys. Rev. D. 1974, vol.10, pp. 275-289.

[26] C. Wolf, "Sub-quark structure and the magnetic moments of the u, d quarks in the Rishon model", Acta Phys. Polo. 1987, vol. B18 (5) 421-427.

[27] P. Lancini and R. Mignani, "The Electron in a (3+3)Dimensional space-time", in V. Simulik Ed. What is electron?, Canada: Apeiron, 2005, pp. 155 - 168.

[28] J. Keller, "A comprehensive theory of the electron from START" in V. Simulik Ed. What is electron? Canada: Apeiron, 2005, pp. 1-28.

[29] H. E. Puthoff, "Gravity as a Zero-Point-Fluctuation Force”. Phys. Rev. A, 1989, vol. 39, no. 5, 2333-2342.

[30] J. D. Jackson, Classical Electrodynamics, Singapore: John Wiley \& Sons (Asia) Pte Ltd., 2007.

[31] P. A. M Dirac, "Classical theory of radiating electron", Proceedings of the Royal Society of London. Series A. Mathematical and Physical Sciences, 1938, vol. 167 , pp. $148-169$.

[32] J. M. Levy-Leblond, "Classical charged particles revisited: renormalizing mass and spin”, Eur. J. Phys, 1989, vol. 10, $265-268$.

[33] N. N. Sirota, "Compressive and centrifugal forces in a rotating charged body and the semiclassical electron radius", Russ. J. Phys. vol. 48, no. 9, 993 - 995, 2005.

[34] G. N. Ramachandran et al., "A semi-classical model of the electron containing tachyonic matter", Phys. Lett., 1972, vol. 39A, pp. $383-384$.

[35] R. A. Lee and J. Liesegang, "A Semi classical picture of the electron spin", Phys. Lett. 1971, vol. 37A, pp 37-38.

[36] L. L. Foldy and S. A. Wouthuysen, "On the Dirac theory of spin 1/2 particles and its non-relativistic limit", Phys. Rev., 1950, vol. 78, pp. 29-36. 
[37] A. G. Nikitin, "On exact Foldy-Wouthuysen transformation”, J. Phys. A. Math. Gen. 1998, vol. 31, pp. 3297-3300.

[38]J. P. Costella and B. H. J. McKellar, "The FoldyWouthuysen transformation", Am. J. Phys. 1995, vol. 63 1119-1121.

[39] M. H. MacGregor, "What causes the Electron to weigh?" in V. Simulik Ed. What is electron? Canada: Apeiron, 2005, pp. 129 - 153.

[40] M. H. MacGregor, “The Power of $\alpha$ ”, Singapore: World Scientific, 2007.

[41] M. Rivas, "The spacetime symmetry group of a spin $1 / 2$ elementary particle", J. Phys. A: Math. Gen., 2006, vol. 39, 4291-4308.

[42] M. Rivas, "The spinning electron”, in V. Simulik Ed. What is electron?, Canada: Apeiron, 2005, pp. 59-82.

[43] M. Rivas, Kinematical formalism of elementary spinning particles, Lecture Notes at Centre for Theoretical Physics, Jamia Milia Islamia Universiy, New Delhi, 2007.

[44] V. Simulik, "The Electron as a system of classical electromagnetic and scalar fields", in V. Simulik Ed. What is electron?, Canada: Apeiron, 2005, pp. 105-127.

[45] A. O. Barut and A. J. Bracken, "Zitterbewegung and the internal geometry of the electron", Phys. Rev. D, 1981, vol. 23, pp. 2454-2463.

[46] A. O. Barut and N. Zanghi, "Classical model of the Dirac electron”, Phys. Rev. Lett., 1984, vol. 52, pp. 2009-2012.

[47] B. G. Sidharth, "Revisiting Zitterbewegung", Int. J. Theor. Phys. vol., 2009, 48, pp. 497-506.

[48] D. Hestenes, "Quantum Mechanics from selfinteraction”, Found. Phys. 1983, vol. 15, pp. 63 - 87.

[49] D. Hestenes, "The Zitterbewegung interpretation of Quantum Mechanics”, Found. Phys., 1990, vol. 20, pp. 12131232 .

[50] D. Hestenes, "Zitterbewegung modelling", Found. Phys., 1993, vol.23, pp. 365-387.

[51] W. A. Rodrigues Jr., J. Vaz Jr., E. Recami and G. Salesi, "About zitterbewegung and electron structure", Phys. Lett. B, 1993, vol. 318, pp. 623--628.

[52] G. David and J. Cserti, "General theory of Zitterbewegung”, Phys. Rev. B, 2010, vol. 81, pp. 121417(R) $1-4$.

[53] S. Rupp, T. Sigg and M. Sorg, "Zitterbewegung and quantum jumps in relativistic Schrodinger theory", Int. J. Theor. Phys., 2000, vol. 39, 1543-1597.

[54] M. Villavicencio and J. A. E. Roa-Neri, "Zitterbewegung in a static and uniform magnetic field, Eur. J. Phys., 2000, vol. 21, pp. 119-123.

[55] N. Hamdan, A. Altorra and H. A. Salman, "Modified Dirac equation with classical zitterbewegung", Proc. Pak. Acad. Sci., 2007, vol. 44, pp. 263-272.

[56] R. C. Jennison, "A new classical relativistic model of the electron", Phys. Lett. A., 1989, vol. 141, pp. 377-382.

[57] F. Rohrlich, "The dynamics of a charged sphere and the electron", Am. J. Phys., 1997, vol. 65, pp. 1051-1056.

[58] A. B. Nassar, "Schrodinger equation for an extended electron”, Int. J. Theor. Phys., 2007, vol. 46, pp. 548-552.

[59] C. L. Pekeris, "On the possibility of a hydrodynamic model of the electron", Proc. Nat. Acad. Sci. USA, 1975, vol. 72, 2037-2040.

[60] O. Gron, "Repulsive gravitation and electron models", Phys. Rev. D, 1985, vol. 31, pp. 2129-2131.

[61] M. Pavsic, E. Recami, W. A. Rodrigues Jr., "Spin and electron structure", Phys. Lett. B., 1993, vol. 318, pp. 481488 .

[62] Z. Grossmann and A. Peres, "Classical theory of the Dirac electron”, Phys. Rev., 1963, vol. 132, pp. 2346-2349.

[63] J. M. Aguirregabiria, A. Hernandez, M. Rivas and M. A. Valle, "On the equilibrium configuration of point charges placed on an ellipse", Comp. in Phys. 1990, pp. 60-63.

[64] S. Ghosh, A. Choudhury and J. K. Sarma, "Radius of Electron, Magnetic Moment and Helical Motion of the charge of electron”, Apeiron, 2012, vol. 19, pp. 247.

[65] T. Aoyama et al. "The anomalous magnetic moment of the muon in the Standard Model", Phys. Rept., 2020, vol. 887 , pp. 1-166.

[66] T. Blum et al., "Hadronic Light-by-Light Scattering Contribution to the Muon Anomalous Magnetic Moment from Lattice QCD”, Phys. Rev. Lett., 2020, vol. 124, pp. 132002-1-6.

\section{Author's biography}

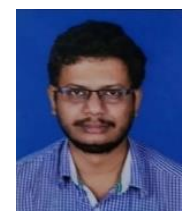

Dr. Sovan Ghosh did his doctoral research on different properties of the electron and aspect of various structural models of the electron. $\mathrm{He}$ is currently working as Assistant Professor in a college of Bangalore, India. 